\title{
QoS MANAGEMENT FOR MOBILE SATELLITE COMMUNICATION
}

\author{
M.Adhirai ${ }^{1}$, R. Kurunjimalar ${ }^{2}$ \\ ${ }^{1} P G$ Scholar, ${ }^{2}$ Associate Professor, ECE Department, SMVEC, Puducherry, India
}

\begin{abstract}
In this paper, a cross-layer architecture (QoSatAr) is developed to provide end-to-end quality of service (QoS) guarantees for Internet protocol (IP) traffic over the Digital Video Broadcasting-Second generation (DVB-S2) satellite systems. The architecture design is based on a cross-layer optimization between the physical layer and the network layer to provide QoS provisioning based on the bandwidth availability present in the DVB-S2 satellite channel. One of the most important aspects of the architecture design is that QoSatAr is able to guarantee the QoS requirements for specific traffic flows considering a single parameter: the bandwidth availability which is set at the physical layer (considering adaptive code and modulation adaptation) and sent to the network layer by means of a cross-layer optimization. The architecture has been evaluated using the NS-2 simulator.
\end{abstract}

Keywords: QoSatAr, DVB-S2, ACM, RQM, DiffServ

\section{INTRODUCTION}

In recent years there has been a tremendous growth in mobile communications and new wireless technologies have rapidly emerged. As demand for communication connectivity "anytime, anywhere, and any way" increases, heterogeneous network design trend will grow faster supporting seamless integration. Satellite communication plays a significant role in supporting such architectures through hybrid Satellites/Wired/Wireless infrastructure. Within the last decades, geostationary (GEO) satellite systems have become an essential asset for Europe and all society. This infrastructure enables us to communicate and send information globally, allowing to reach large and disperse populations around the world, it makes feasible the provisioning of on-demand data and any type of Internet protocol (IP)-based services in real time.

The main challenges that this technology faces in the provisioning of end-to-end (E2E) QoS guarantees are related to its native characteristics. For instance, the delay, that affects the performance of the transmission control protocol (TCP) [1], can seriously affect the delivery of time critical data to end users. In this article we propose QoSatAr, a cross-layer QoS SATellite ARchitecture to provide E2E QoS guarantees for IP traffic over the forward satellite channel. The architecture design is based on a cross-layer optimization between the physical layer and the network layer to enhance QoS provisioning when different levels of link capacity are available in the satellite system. The design is developed in compliance with the recent standard developed for the Digital Video Broadcasting-second generation (DVB-S2) [2] forward channel. In addition, the architecture is characterized by the separation between higher layers or satellite-independent (SI) layers and lower layers or satellite-dependent (SD) layers. This modular reference architecture allows enhanced control functions performed by the SI layers which can be either modified or updated regardless of the SD layer technology. In this way, the design of the QoSatAr architecture is developed at the SI layers to establish priorities among users and applications (allocated at higher layers) that share the satellite link interface. Here, the interaction with the lower layers is defined in order to encompass the service categorization and the overall performance of the satellite network. Focusing on the SI layers, the management and control functions performed at upper layers [3] are enhanced while the SD layers (i.e., satellite physical, MAC, and link control which are strictly satellite dependent) are isolated to include different physical layer supports (i.e., for heterogeneous networks).

On the other hand, the DVB-S2 standard defines as mandatory the use of the adaptive code and modulation (ACM) [4] techniques, to attain Interactive Services. Such techniques reduce the available link bandwidth (transmission rate), if necessary, to achieve quasi-error-free channel conditions for each individual user to provide them with the most suitable Modulation and Code (ModCod) value according to the measured signal-to-noise-plus interference-ratio (SNIR) value reported by the return channel. The major benefit of adopting ACM techniques it is that the obtained spectral efficiency is optimized, being as high as possible for all the satellite terminals. Nevertheless, there is a fundamental change related to the satellite physical layer as it is considered constantly changing. In this way, one of the main concerns using GEO satellite systems is the management of these bandwidth variations to satisfy the specified QoS levels for different traffic classes. 
The QoSatAr design is developed inside the DVB-S2 gateway which is the central element in the architecture. This is done in order to allow satellite operators to easily adopt the proposed architecture with low deployment cost.

\subsection{DiffServ and IntServ [5]}

The Internet is being used by business and user communities with widely varied service expectations from the network infrastructure. For example, many companies rely on the Internet for the day-to-day management of their global enterprise. These companies are willing to pay a substantially higher cost for the best possible service level from the Internet. Similarly, there are many users who are willing to pay a higher Internet access fee in order to make use of demanding applications, such as IP telephony and videoconferencing. At the same time, there are millions of users who want to pay as little as possible for more elementary services, like exchanging e-mails and/or surfing the Web. In addition to this variety of user expectations, there has also been a rapid evolution in the set of Internet applications. A few years ago the key Internet applications were only e-mail, ftp, or newsgroups.

In contrast, the present-day Internet applications have widely diverse service needs because they transfer a wide range of information types, including voice, music, video, graphics, Java scripts, and hypertext links. As a result of these changes in user expectations and Internet applications, there is a growing demand to replace the current same-service-to-all paradigm with a model in which users, applications, or individual packets are differentiated based on their service needs. Architectures for providing service differentiation in the Internet have been the focus of extensive research in the last few years. These research efforts have identified two fundamentally different approaches for service differentiation: integrated services and differentiated services.

\section{E2E QoSatAr ARCHITECTURE DESIGN}

The QoSatAr design includes

(i) A cross-layer optimization between the physical layer and the network layer to provide E2E QoS guarantees, considering the fact that the DVB-S2 forward channel is affected by the presence of rain events.

(ii) A complete active queue management (AQM) system that considers Token Buckets (TBs) as rate limiters to regulate and guarantee a minimum transmission rate for each traffic class according to the priority levels established by the satellite operator. Here, the queue design considers the bandwidth delay product (BDP) value to dynamically set the queue lengths to enforce bounded delay values for high-priority traffic classes.

(iii) A modified queuing policy called re-queuing mechanism (RQM) to reduce delay and jitter while improving the user's Quality of End user (QoE) for the expedited forwarding (EF) and the Assured Forwarding (AF) traffic classes. This mechanism follows the philosophy of the DVB-S2 design, in which retransmissions are avoided because the two-way propagation delay is significantly high. In our case, the RQM mechanism prevents dropping packets that do not fulfill the DiffServ traffic class specification.

(iv) A dynamic IP scheduler to allocate bandwidth resources for prioritizing those flows with high QoS requirements. The IP scheduler uses an algorithm that adjusts its internal values considering the capacity present in the system. This dynamic adaptation considers the cross-layer information sent by the physical layer to provide enhanced priority for specific flows when a reduced and limited channel capacity is experienced in the satellite system.

\subsection{E2E QoSatAr Architecture}

This section describes the main functional blocks inside the gateway for the provisioning of E2E QoS guarantees over a DVB-S2 satellite system. The QoSatAr architecture is designed based on the DiffServ framework to provide E2E QoS guarantees. The DiffServ architecture defined by the IETF allows IP traffic to be classified into a finite number of classes differentiated by priority, to support different QoS levels. The main components defined in the DiffServ architecture are the traffic classifiers, which select packet and assign (if necessary) their differentiated services code point (DSCP) values; the traffic conditioners which mark and enforce the rate limitation policy; and the Per Hop Behavior (PHB) that enforces the differentiated packet treatments. In this sense, there are three predefined PHBs: EF, AF, and besteffort (BE). One of the main benefits of adopting the DiffServ framework is that the network complexity is translated to edge nodes, enabling to maintain the scalability and simplicity of the IP network.

The proposed QoSatAr gateway design is focused on the SI layers, in order to empower the QoS functions performed at this layer, while isolating the SD layers (i.e., satellite physical, MAC, and link control) to include different physical layer supports (for heterogeneous networks) [6]. The proposed design including its separation between high SI layers and low SD layers is shown in Fig 1. Particularly, the SI layers are defined to deal with QoS differentiation based on the DiffServ framework. Conversely, the SD layers are proposed for applying different DVB-S2 channel adaptations. 


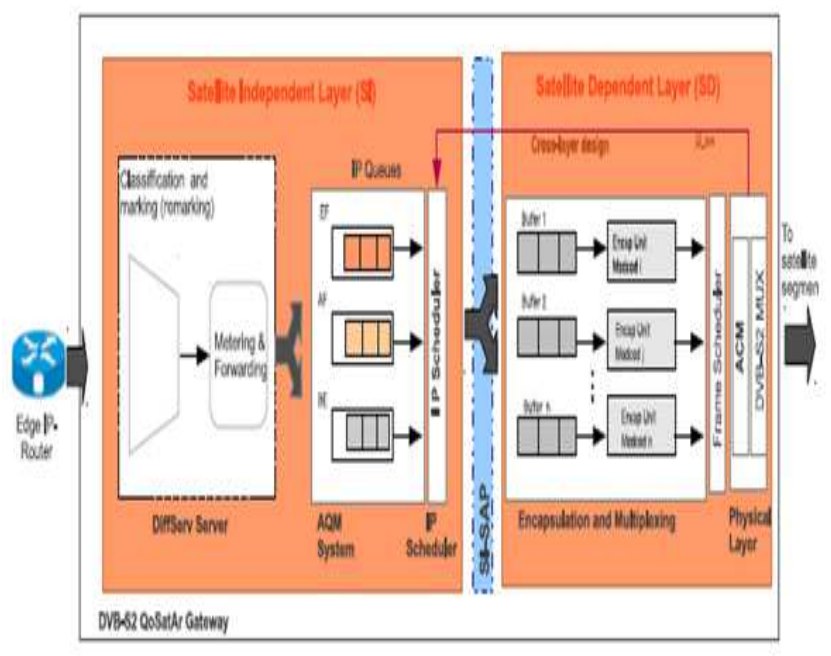

Fig: 1 DVB-S2 QoSatAr gateway design

The QoS blocks and their functionalities as a part of the proposed cross-layer QoSatAr architecture are described as follows: at the SI layer, the DiffServ server, the AQM system, and the IP scheduler are set up. At the SD layer, several buffers are defined for allocating packets waiting to be encapsulated and multiplexed before these are forwarded to the required RCST. For simplicity at SI layers, we have reduced the set of DiffServ traffic classes into three (EF, AF, and BE). Similarly, at the SD layers several queues are set which are associated with different ModCods schemes. In this scenario, ModCodi is said to have higher spectral efficiency than ModCodj (where $\mathrm{i}<\mathrm{j}$ ), we assume that ModCods are ordered from high to low spectral efficiency. This design complies with the ETSI-BSM-QoS functional architecture supported by the standards ETSI TS 102157 and ETSI TS 102 462.

\subsection{SI Functional Blocks}

In order to determine the QoS treatment to be applied through the satellite network, packets coming from other IP networks are marked. In most of the cases, packet marking is typically performed at the edge nodes of the DiffServ satellite domain. However, in some cases satellite operators may require a remarking process to be performed inside the gateway to adjust the forwarding policy that will be applied.

As it is shown in Fig 1, packets entering the DVB-S2 QoSatAr gateway are received by the DiffServ server. This module is responsible of receiving different flows, classifying the incoming packets, and deciding (if required) if a packet needs to be re-assigned with a different QoS level, by marking/remarking its DSCP. In practice, the gateway implements packet classification and per hop forwarding scheduling according to the DSCP value of each packet. At this point, packets are forwarded to get in the AQM system.

\subsubsection{The AQM System}

The detailed design inside the AQM system is depicted in Fig 2. Notice that the proposed scheme allows multiple flows to be aggregated and treated as a single flow per traffic class. Packets coming from these queues are scheduled to the SD layers based on a dynamic IP scheduler. Here, IP scheduler functions are linked intrinsically with the satellite bandwidth allocation carried out by lower layers.

As it is observed in Fig 2, the high-priority traffic classes (EF and $\mathrm{AF}$ ) implement TBs as rate limiters to guarantee certain transmission rates for each traffic class. These rates are defined in accordance with the bandwidth assignment established in the SLAs between the satellite operator and the subscribers. Importantly, the operator is able to modify these rules as a part of the operation and management tasks in the satellite gateway. The incoming TBs limiting rates represent the transfer rates for the EF and AF traffic classes ( $\mu \mathrm{EF}$ and $\mu \mathrm{AF}$, respectively). Using $\mathrm{TBs}$, packets are separated in two levels: in-profile (fulfill the SLA) and out-of-profile packets (do not fulfill the SLA). Alternatively, the BE traffic class is not provided with a TB policer, this assumption is made considering that this traffic type does not need to adjust a committed rate.

Therefore, when the BE queue is full, no particular algorithms are needed to decide which packet is going to be dropped. As a result, a simple drop tail (DT) mechanism is implemented. The QoS policy implemented in the QoSatAr architecture allows all in-profile packets (coming from the $\mathrm{EF}$ and $\mathrm{AF}$ traffic classes) to be sent directly to the IP scheduler. This element is defined to control the order in which packets are extracted out from its queues. Here, it is important to bear in mind that the IP scheduler incoming rate is limited by each TB.

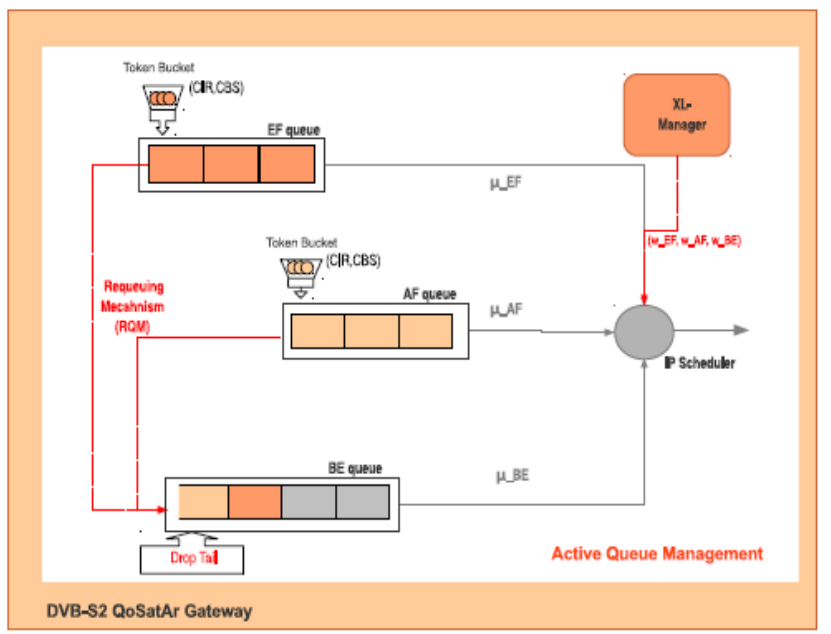

Fig. 2 the AQM mechanism design inside the gateway 


\subsubsection{The RQM Mechanism}

In the same scenario of Fig. 2, when the TB algorithm runs out of tokens, the out-of-profile EF and AF packets are detected; this means that the sender generates more packets per time unit than the packets allowed by the SLA. For fixed networks with a relatively low RTT, the general recommendation is to drop these out-of-profile packets [23]. Nevertheless, the situation over the DVB-S2 satellite link is different, because this type of link virtually does not loose packets, given that the RTT is high. In this condition, we propose not to drop the outof-profile EF and AF packets, but instead send them to be enqueued again but in this case through the $\mathrm{BE}$ queue. We have called this modified queuing mechanism as the RQM (also shown in Fig 2).

The main objective with the adoption of the RQM mechanism is to reduce the latency and jitter experienced by the userapplication for the EF and the AF traffic classes. Given that our model does not drop these out-of profile packets, but instead these packets are sent to the BE queue. This modified RQM mechanism allows the out-of profile packets to downgrade its QoS level, giving them the possibility (in the case of TCP) to reach the receiver before an RTT. The downgraded packet will get in the destination later than the other transmitted packets, since this downgraded packet will be re-classified to the BE traffic class. This packet disorder will be detected at the sender side by means of the TCP's triple dupACK mechanism. Therefore, the TCP will trigger the fast retransmit/ fast recovery algorithm as a congestion control signal.

As a response the sender will reduce its congestion window, forcing to fulfill the SLA. It is worth mentioning that in this model the input traffic of the BE traffic class is not totally independent from its higher-priority traffic classes. Conversely, if the protocol used to transport the high priority traffic classes is different to the TCP protocol, the ability to detect out-of-order delivery will depend on the upper-laying protocol (UDP/RTP/RTCP) used to reconstruct the sender's packet sequences at the destined user application.

\subsubsection{The QoSatAr Queue Design}

The design of the QoSatAr architecture requires setting the queue length for each specific traffic class in order to keep the delay bounded for the high-priority traffic classes. To do so, we express the system load (the number of packets sent but not yet acknowledged) for each Diffserv traffic class $i$ at time $t$ as

$$
\operatorname{Li}(t)=\mu i(t) \cdot \operatorname{RTTmin}+\beta i(t)=\operatorname{BDPi}(t)+\beta i(t) \quad---(1)
$$

Where $\operatorname{Li}(\mathrm{t})$ represents the system load, $\mu \mathrm{i}(\mathrm{t})$ represents the TB's limiting rates for each high-priority traffic class i $(\mu \mathrm{EF}$ and $\mu \mathrm{AF}$ ), RTTmin represents the two-way propagation delay or minimum RTT (set to $560 \mathrm{~ms}$ in the GEO satellite scenario). $\beta \mathrm{i}(\mathrm{t})$ is the queue occupancy level and $\mathrm{Bi}$ is the queue size.

Here, the maximum system load available for each traffic class $i$ at time $t$ is

$$
\operatorname{Lmax} i(t)=\mu i(t) \cdot R T T m i n+B i---(2)
$$

Therefore, the queue length and the occupancy queuing level for the $\mathrm{EF}$ and $\mathrm{AF}$ traffic classes are set to their BDP values:

$$
\mu \mathrm{i}(\mathrm{t}) \cdot \mathrm{RTTmin}=\mathrm{BDPi}(\mathrm{t})=\mathrm{Bi}----(3)
$$

Basically in the QoSatAr, the TBs limiting rates are used to regulate the queue occupancy level to enforce the system to work at a reference load level. This reference working point is a function of the defined SLAs for each DiffServ traffic class. By considering this queue design, it is possible to keep an optimal operation-working point while enhancing the satellite efficiency, given that the amount of packets to be buffered in the AQM system is set equal to the total in-flight packets that the satellite system is able to transport. Finally, when all packets have been queued and processed by the AQM module (see Fig 2), they are sent directly to the IP scheduler.

\subsubsection{The adaptive IP Scheduler}

The IP scheduler design has an important impact on providing E2E QoS guarantees, mainly, because the IP scheduler defines how the gateway allocates the bandwidth capacity to those flows requiring higher QoS guarantees, at the forward channel. In the QoSatAr, the IP scheduler (which is the highest hierarchical scheduler) is responsible for queuing IP packets in the dedicated ModCod queues, providing them with QoS differentiation while tracking channel variations to determine the applicable ModCod for each forwarded packet. To provide QoS differentiation among Diffserv queues, the proposed IP scheduler dynamically adapts its values to determine the number of extracted packets every time it visits a queue. It is based on the Weighted Round Robin (WRR) mechanism in which a weight adaptation is performed considering the bandwidth availability present in the satellite system. This design allows to provide QoS guarantees among DiffServ flows taking into account the link bandwidth variations reported by the physical layer. The adaptive IP scheduler design is shown in Fig 3. 


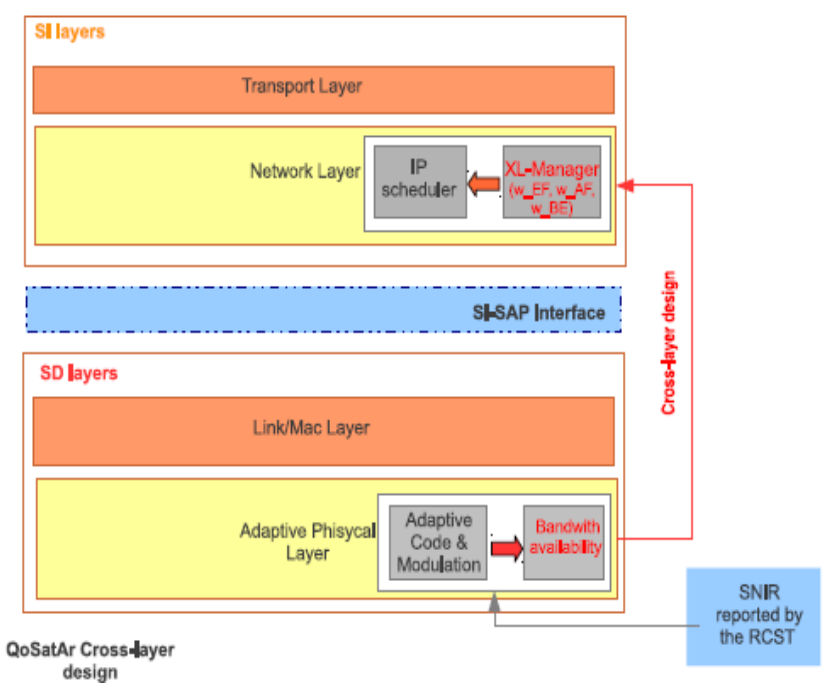

Fig. 3 The cross layer design for IP scheduler

As it is observed, along with the IP scheduler, there is a new module responsible for calculating the weight values. This component is referred as the Cross-layer (XL) manager that takes into account the bandwidth availability to compute the suitable weight values for each traffic class (WEF, WAF, and WBE). It considers as an input parameter the bandwidth availability reported by the physical layer to enhance the QoS provisioning among flows. This information is sent from the physical layer to the network layer based on a cross-layer optimization. The cross-layer design for the adaptive IP scheduler is also shown in Figure 3. Here, the corresponding weight values are set within the XL manager to prioritize the resources for the high priority traffic classes. As it is observed, this module is decoupled from the IP scheduler; therefore, the scheduler complexity is not increased and both modules can work independently based on their own settings.

One of the main contributions of the QoSatAr architecture is that the IP scheduling algorithm is able to guarantee the QoS requirements for specific traffic flows using a single parameter: the bandwidth availability. This parameter is set at the physical layer (considering the ACM adaptation) and sent to the IP scheduler by means of a cross-layer design.

\subsubsection{Satellite Bandwidth Characterization}

The recent standards developed for the DVB-S2/RCS physical layer define as normative the use of the ACM techniques to attain Interactive Services. One of the main advantages of using the ACM techniques is the ability to achieve quasi-errorfree channel conditions for each individual user, by providing them with the most suitable ModCod value according to the measured SNIR, so that the spectral efficiency is as high as possible in all the cases. To do so, the DVB-S2 physical layer takes advantage of the SNIR value reported by each RCST.

\subsection{SD Functional Blocks}

The SD layer design inside the gateway is shown in Fig 3.1. As it is observed, packets are sent through the SD layer (after scheduling functions) using the QoS mapping concept. This implements Queue Identifiers (QID) to send the DiffServ flows from the SI layers to the SD layers. This procedure is allocated at the Satellite Independent-Service Access Point interface, which follows the guidelines defined in [6]. At this point, each QID enables the IP packet to be allocated to a virtual queue (considering its QoS characteristics) and then transported across the SD layers. For simplicity, the QoS mapping between SI and SD layers is not detailed in Fig 1.

The SD layer design includes a set of physical buffers, their associated encapsulation units, a Frame Scheduler, and a DVB-S2 multiplexer. These elements are used to construct frames using IP packets and transmit them through the DVBS2 physical layer to those destination terminals that have similar propagation conditions. In particular, when enough packets are stored in a Mod- Cod queue, the encapsulation units are used to build DVB-S2 frames which are served by the Frame Scheduler to feed the satellite physical layer (see Fig 1). According to the DVB-S2 frame structure, frames coming from different encapsulating units are transmitted through the satellite link using different ModCods. The corresponding ModCod is determined by the terminal that is under the worst propagation conditions, assuming that all destination terminals (in a beam) have similar propagation conditions. As a consequence, the frames sent to terminals having good propagation conditions (i.e., in clear sky) will be queued to encapsulation units using a ModCodi that provides low bit protection. Conversely, the frames sent to terminals having bad propagation conditions (i.e., facing a rain event) will use a Mod-Codn with higher bit protection levels, thus requiring additional overhead.

To guarantee a maximum waiting time for each IP packet, the QoSatAr frame scheduler follows a strategy based on the queue status. In this way, if an IP packet has reached its maximum waiting time and it is still waiting in the queue (of a certain encapsulation unit), the frame scheduler should encapsulate this IP packet into the next DVB-S2 frame and transmit it through the satellite link. However, if the minimum frame size is not reached, several IP packets from other encapsulation unit (having different ModCodj that require less protection) must be used to fill the frame size. As a result it is possible to assure the maximum waiting time for each IP packet. Finally, to guarantee fairness among terminals, the QoSatAr design (as most of the broadcast systems) is based on the TDM sharing policy with the adoption of ACM techniques. In this context, terminals under good propagation conditions are able to use ModCods with lower overhead increasing their transmission rates compared to those terminals under bad weather conditions. For this reason in practice, the fairness policy applied to the DVB-S2 frame scheduler tries to 
shield the network layer from the effects of a time and location dependent physical layer.

In this way, using ACM techniques it is possible to select the proper ModCod to guarantee a determined (low) error probability. Therefore, to offer homogeneous service among terminals, the DVB-S2 frame scheduler approximately assigns the same shared-service rate regardless of terminal's propagation conditions. As a result, the offered transmission rate is the same, while the time used to transmit frames depends on the propagation conditions for each destination terminal. Notice that if the frame scheduler implements other policy like sharing the same amount of transmission time among terminals, it will penalize the terminals under the worst propagation conditions, so we would have a similar situation to what happen within DVB-S.

\section{PERFORMANCE EVALUATION}

In this section, the proposed QoSatAr architecture is evaluated using the NS-2 simulation tool. Here, we describe the general satellite settings used to conduct the simulation tests, including the performance metrics to evaluate and compare the simulation results. We provide the results of evaluating the QoSatAr architecture working in a BSM satellite system is provided.

In this section, the proposedQoSatAr architecture is evaluated using the NS-2 simulation tool. Here, we describe the general satellite settings used to conduct the simulation tests, including the performance metrics to evaluate and compare the simulation results. In addition, we provide the results of evaluating the QoSatAr architecture working in a BSM satellite system. The EF class supports a real-time VoIP application, simulating a constant-rate traffic, which is transferred over the user data protocol (UDP) to strictly guarantee bandwidth reservation. The AF traffic class bears a HTTP application while the BE traffic class bears a persistent FTP transaction server. Both, the AF and BE traffic classes are transported using the TCP protocol.

The DVB-S2 satellite environment is simulated using the Linux implementation for NS-2 version 2.29. The QoSatAr architecture has been integrated in the NS-2 simulator using the DiffServ module provided in [7]. Particularly, the functionalities of the RQM mechanism and the dynamic IP scheduler have been added in the code to test the capability of the proposed architecture. The objective of this simulated scenario is to conduct a performance evaluation of the QoSatAr architecture in the presence of bandwidth variations.

To evaluate the impact of adopting the QoSatAr architecture working over the proposed satellite scenario, we initially perform a simulation test to evaluate the benefits of using the RQM mechanism instead of using a simple DT.
The metrics used to evaluate the performance of the selected TCP variants working over the proposed DVB-S2 ETSI-BSM QoS scenario are the goodput, the queue occupancy, the delay, and jitter. It is important to bear in mind that performance analysis is carried out over the DVBS2 satellite forward link so that the performance metrics are defined considering the information measured in this link.

Fig 4 illustrates the total received $\mathrm{AF}$ packets at the application layer. As it is shown, when using the proposed RQM mechanism during all the simulation time. In contrast to the DT scheme in which the levels of received packets are less. Therefore, using RQM, the number of correctly received packets have increased in $44 \%$ (in average) compared with the DT scheme. This result is caused mainly because when using the proposed RQM mechanism, the out-of-profile AF packets are not dropped.

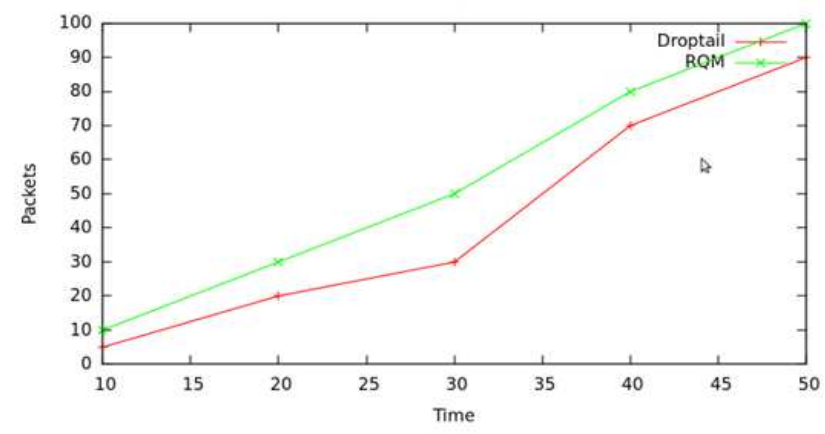

Fig.4 Total received AF packets

Fig. 5 shows similar simulation results for the EF traffic class using both queuing options: either the RQM or the DT mechanism. Here, the same performance parameters have been evaluated. As it is observed, when using the proposed RQM to transport the EF traffic class (using UDP protocol), similar results have been obtained compared to the AF traffic class. The obtained results enable us to conclude that when the RQM mechanism is employed, the EF and AF goodput levels are improved.

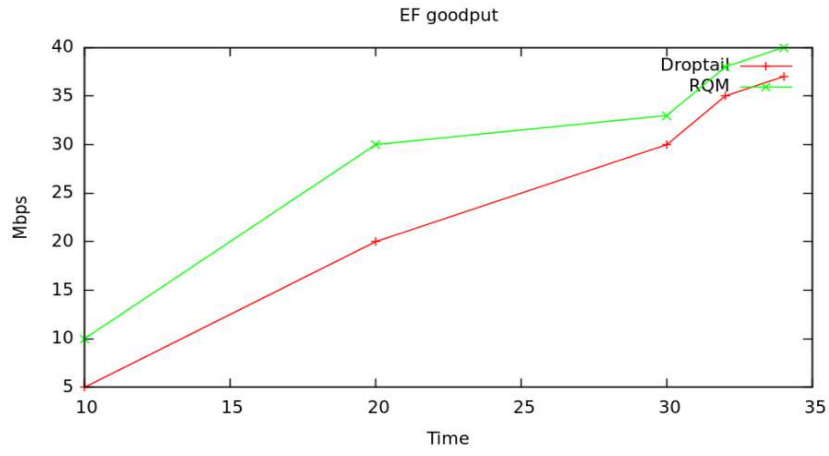

Fig. 5 EF throughput 


\section{CONCLUSIONS}

In this paper, we have presented QoSatAr, a cross-layer architecture developed to provide E2E QoS guarantees for IP traffic over the DVB-S2 satellite channel. The architecture design is based on a cross-layer optimization between the physical layer and the network layer to provide QoS provisioning based on the bandwidth variability present in the satellite system. Our design is developed at the SI layers, being in compliance with the ETSI-BSM-QoS standards.

In this paper, we have detailed a complete QoS design inside the gateway, in which we have proposed the RQM mechanism to enhance the goodput for the EF and AF traffic classes while reducing the E2E delay and jitter. In addition, we have proposed an adaptive IP scheduler to guarantee the highpriority traffic classes regardless of the channel condition affected by rain events.

The implementation of this architecture has been evaluated using the NS-2 simulator. The key results allow us to confirm that using QoSatAr, it is possible to keep control of the satellite system load while guaranteeing QoS levels for the high-priority traffic classes even though bandwidth variations due to rain events are experienced. The simulation results also demonstrate that with the adoption of the proposed the RQM mechanism, the user's QoE is improved while keeping bounded delay and jitter values for the high-priority traffic classes.

\section{REFERENCES}

[1]. C Caini, R Firrincieli, M Marchese, T de Cola, M Luglio, C Roseti, N Celandroni, F Potort 1 , Transport layer protocols and architectures for satellite networks, Int. J. Satellite Commun. Netw. 25, 1-26 (2007)

[2]. 300421 EE, ETSI Digital Video Broadcasting (DVB); Second Generation. Framing structure, channel coding and modulation system for 11/12 Ghz Satellite Services 1997. [ETSI Specification: ETSI EN 300 421]

[3]. M Marchese, M Mongelli, in IEEE ICC Proceedings, Beijing (China) Protocol structure overview of QoS mapping over satellite networks. (2008), pp. 1957-1961, doi:10.1109/ICC.2008.375

[4]. R Rinaldo, M Vazquez-Castro, A Morello, DVB-S2 ACM modes for IP and MPEG unicast applications, Int. J. Satellite Commun. Netw. 22, 367-399 (2004)

[5]. C Dovrolis, P Ramanathan, A case for relative differentiated services and the proportional differentiation model, IEE Netw. 13(5), 26-34 (1999)

[6]. M Marchese, M Mongelli, in IEEE ICC Proceedings, Beijing (China) Protocol structure overview of QoS mapping over satellite networks. (2008), pp. 1957-1961, doi:10.1109/ICC.2008.375

[7]. P Pieda, J Ethridge, M Baines, F Shallwani, in Open IP, Nortel, Networksinternal document A network simulator differentiated services implementation, 2000

\section{BIOGRAPHIES}

M. Adhirai is a PG Scholar in Electronics and Communication at Sri Manakula Vinayagar Engineering College and she pursued her UG from Arunai Engineering College, Tiruvannamalai, India.

R. Kurunjimalar, working as an Associate Professor, Electronics and Communication Engineering, SMVEC. 